\title{
Distribution of Salmonella pathogenicity island (SPI)-8 and SPI-10 among different serotypes of Salmonella
}

Correspondence

Jayant R. Bandekar

jrb@barc.gov.in

Received 13 September 2007

Accepted 3 January 2008

\author{
Sunil D. Saroj, R. Shashidhar, Manisha Karani and Jayant R. Bandekar
}

Food Technology Division, Bhabha Atomic Research Centre, Mumbai 400 085, India

\begin{abstract}
Many virulence phenotypes of Salmonella enterica are encoded by genes located on pathogenicity islands. Based on genome analysis, it is predicted that Salmonella pathogenicity island (SPI)-8 is restricted to Salmonella serovars Typhi and Paratyphi A, and SPI-10 to Salmonella serovars Typhi, Paratyphi, Enteritidis, Dublin and Gallinarum. This study was conducted to investigate the distribution of SPI-8 and SPI-10 among Salmonella isolates from sprouts, fish, water and blood. A total of 110 Salmonella isolates and 6 Salmonella serovars from the Microbial Type Culture Collection, Chandigarh, India, were screened. All isolates belonging to Salmonella serovars Washington, Enteritidis and Paratyphi A had both SPI-8 and SPI-10. All Salmonella serovar Typhi isolates from water and blood had both SPI-8 and SPI-10, whereas isolates from fish contained only SPI-8. SPI-8 and SPI-10 were also detected in only 3 out of 42 isolates belonging to Salmonella serovar Typhimurium. Both SPI-8 and SPI-10 were absent in Salmonella serovars Worthington, Dublin, Paratyphi B and Paratyphi C. These results contradict the predictions from Salmonella genome sequences available in GenBank and indicate that SPI-8 and SPI-10 are widely distributed among Salmonella serovars and that virulence factors other than those on SPI-8 and SPI-10 may be responsible for host specificity. This is the first report on the distribution of SPIs in Salmonella isolates from India.
\end{abstract}

\section{INTRODUCTION}

The genus Salmonella contains pathogens that are closely related genetically but differ in their host range (Baumler et al., 1998). Salmonella enterica contains more than 2500 serovars. Serovar Typhimurium causes systemic disease in mice, but may colonize other species such as pigs, poultry, horses, cattle, and sheep, and can cause gastroenteritis in humans, whereas serovar Typhi is a prototypical hostrestricted serovar that causes typhoid fever in humans and higher primates, but is unable to produce illness in other vertebrate species (Townsend et al., 2001). Large numbers of Salmonella outbreaks have been reported as a result of the consumption of fresh, minimally processed foods, poultry meat, contaminated water and undercooked eggs (BairdParker, 1990; Lynch et al., 2006; Plym-Forshell \& Wierup, 2006). Salmonella contamination of different food products

Abbreviations: PAl, pathogenicity-associated island; SPI, Salmonella pathogenicity island.

The GenBank/EMBL/DDBJ accession numbers for the SPI-8 and SPI10 sequences of the Salmonella isolates determined in this study are EF033079-EF033108.

A table of sequences of the primers used in this study and figures showing DNA dot blots for SPI-8 and SPI-10 and the molecular typing of Salmonella serovars are available as supplementary material with the online version of this paper. such as fish, vegetables, fruit and sprouts has been reported in India (Bandekar et al., 2004; Dhokane et al., 2006; Saroj et al., 2006).

Many of the virulence phenotypes of S. enterica are encoded by genes on pathogenicity-associated islands (PAIs). PAIs consist of large regions of genomic DNA (approx. 10$200 \mathrm{~kb}$ ) that are present in pathogenic bacterial strains but absent from the genomes of non-pathogenic members of the same or related species (Wilson et al., 2002). PAIs are genetic elements on the chromosomes of a large number of pathogens and are considered to produce quantum leaps in bacterial evolution (Groisman \& Ochman, 1996; Schmidt \& Hensel, 2004). The acquisition of PAIs by horizontal gene transfer enables bacteria to gain complex virulence functions rapidly from other species. PAIs on Salmonella are referred to as Salmonella pathogenicity islands (SPIs). At present, 17 different SPIs have been described that encode the most prominent virulence phenotypes, i.e. host-cell invasion and intracellular pathogenesis (Hensel, 2004; Chiu et al., 2005; Vernikos \& Parkhill, 2006).

Based on the genomic data available, Hensel (2004) predicted that SPI-8 would be restricted to Salmonella serovar Typhi and SPI-10 to serovars Typhi and Enteritidis. Whole-genome analysis has shown the presence of SPI-8 in serovars Choleraesuis and Paratyphi A as well as in serovar 
Typhi. In addition, a BLAST search across the current GenBank database has revealed that SPI-10 is present in Salmonella serovars Typhi, Paratyphi A, Enteritidis, Dublin and Gallinarum. The identification and distribution of PAIs in different Salmonella serovars is essential in understanding the development of disease and the evolution of bacterial pathogenesis. Although Salmonella infections are endemic in India, no database on genotypic characteristics of Salmonella isolates from India exists. Therefore, detailed characterization of these food isolates with respect to their pathogenicity genes is vital from a public health point of view.

The purpose of this study was to characterize Salmonella isolates from sprouts, fish, water and blood with respect to pathogenicity-related genes on SPI-8 and SPI-10.

\section{METHODS}

Bacterial strains. All standard cultures were obtained from the Microbial Type Culture Collection (MTCC), Chandigarh, India. Salmonella serovar Typhimurium MTCC 98, Salmonella serovar Typhi, Salmonella serovar Paratyphi A, Salmonella serovar Paratyphi $\mathrm{B}$ and Escherichia coli ATCC 35218 were used to standardize PCR conditions. E. coli ATCC 35218, Salmonella serovar Bovismorbificans MTCC 1162, Salmonella serovar Brunei MTCC 1168, Salmonella enterica subsp. arizonae MTCC 660, Salmonella serovar Virchow MTCC 1163, Salmonella serovar Weltevreden MTCC 1169 and 110 Salmonella isolates (18 blood, 39 water, 31 fish and 22 sprout) were used for screening of SPI-8 and SPI-10. Salmonella isolates from sprouts and fish were from India, whilst water and blood isolates were from Nepal (Bhatta et al., 2007).

Serotyping of the isolates. Antisera (SIFIN) against Salmonella serovars Typhi, Paratyphi A, Enteritidis and Typhimurium were used for serotyping as instructed by the manufacturer.

Molecular typing of Salmonella isolates. Multiplex PCR was carried out using primers for the tyv, fliC-d and viaB genes as described by Hirose et al. (2002) for identification of Salmonella serovar Typhi. To identify Salmonella serovar Enteritidis, PCR was carried out using sdfI primers as described by Agron et al. (2001).

DNA amplification. Gene sequences for SPI-8 and SPI-10 were obtained from GenBank. Regions unique to Salmonella were determined using a BLAST search. Primers were designed using vector NTI software (Infor Max). The primer sequences are given in Supplementary Table S1 (available in JMM Online). The PCR was carried out in a $25 \mu 1$ volume containing $10 \mathrm{mM}$ Tris/ $\mathrm{HCl}(\mathrm{pH} 8.3), 50 \mathrm{mM} \mathrm{KCl}, 1.5 \mathrm{mM}$ $\mathrm{MgCl}_{2}, 1 \mu \mathrm{l}$ primer pairs (10 pmol each), $0.5 \mu \mathrm{l}$ (1.5 U) Taq polymerase, $0.2 \mathrm{mM}$ dNTPs (Bangalore Genie) and $1 \mu$ genomic DNA (200 pg). The PCR was carried out under the following conditions: 30 cycles with heat denaturation at $94{ }^{\circ} \mathrm{C}$ for $30 \mathrm{~s}$, primer annealing at $60{ }^{\circ} \mathrm{C}$ for $30 \mathrm{~s}$ and DNA extension at $72{ }^{\circ} \mathrm{C}$ for $2 \mathrm{~min}$, with a $10 \mathrm{~min}$ final extension at $72{ }^{\circ} \mathrm{C}$, using a programmable thermal controller (Eppendorf AG).The amplified DNA was separated by $1.5 \%$ agarose gel electrophoresis, stained with ethidium bromide and visualized by UV transillumination. Sequencing of amplified products was carried out at Bangalore Genei.

\section{RESULTS AND DISCUSSION}

SPI-8 is a $6.8 \mathrm{~kb}$ region located adjacent to the pheV tRNA gene and SPI-10 is a large insertion of $32.8 \mathrm{~kb}$ located at
tRNA leuX on the genome of Salmonella serovar Typhi (Hensel, 2004). The regions specific for Salmonella were determined using BLASTN at the National Center for Biotechnology Information website (www.ncbi.nih.gov/ BLAST/). Two different sets of primers for SPI-8 (bacteriocin pseudogenes) and three different sets of primers for SPI-10 (Salmonella sefC, sefR and sefB) were designed using these regions. The primers were specific for SPI-8 and SPI10 ; there was no non-specific amplification when tested with E. coli. DNA products of 132 and $142 \mathrm{bp}$ were obtained for SPI- 8 and of 668,625 and 535 bp for SPI-10. The possibility of false negatives was ruled out using a DNA dot-blot assay and that of false positives by sequencing of the PCRamplified products. The results of the DNA dot blot (Supplementary Fig. S1, available in JMM Online) and sequencing of the PCR-amplified products of both SPI-8 and SPI-10 were in accordance with the PCR results. The serotypes of the isolates that showed the presence/absence of SPI-8 and SPI-10 deviating from the BLAST results from GenBank were further confirmed by in-house serotyping using antisera from Germany and by molecular typing (Supplementary Fig. S2 in JMM Online). Based on hybridization studies and comparison of known DNA sequences, the mean DNA sequence similarity among Salmonella serovars is between 96 and $99 \%$ (Edwards et al., 2002). One of the prevailing questions in Salmonella research today concerns the identification of genetic factors that confer on different Salmonella serovars their ability to colonize and in some cases to cause disease in a wide variety of animal hosts (Chan et al., 2003). The pattern of the presence or absence of virulence genes had been used to identify and characterize bacterial isolates using DNA and protein microarrays (Al-Khaldi \& Mossoba, 2004). The main difference between non-pathogenic and pathogenic bacteria of the same species or closely related species is the presence of additional genes in the pathogenic bacteria (Oelschlaeger \& Hacker, 2004). Virulence functions encoded by certain PAIs are lost with a frequency that is higher than the normal rate of mutation. Genetic analyses have shown that such mutations are caused not by defects in individual virulence genes within the PAI, but rather by loss of large regions of the PAI or even the entire PAI (Schmidt \& Hensel, 2004). Therefore, to get information on the complete PAI, two different regions of SPI-8 and three different regions of SPI-10 were analysed by PCR.

SPI-8 was found to be present in all Salmonella isolates belonging to serovars Typhi, Enteritidis, Washington and Paratyphi A, and was absent in serovars Paratyphi B, Paratyphi C, Worthington and Dublin. It was absent in Salmonella serovar Typhimurium isolated from sprouts and fish, but was present in three out of 14 isolates from water (Table 1). These results contradict the predictions from the available genomic data, which indicated that SPI8 would be present only in serovars Typhi and Paratyphi A and not in serovars Enteritidis and Typhimurium.

Townsend et al. (2001) have shown that the distribution of SPI-10 is restricted to Salmonella serovars Typhi, Paratyphi A, 
Table 1. Distribution of SPI-8 and SPI-10 among Salmonella isolates

\begin{tabular}{|c|c|c|c|c|c|c|}
\hline \multirow[t]{2}{*}{ Source } & \multirow[t]{2}{*}{ Salmonella serovar ${ }^{*}$} & \multicolumn{2}{|c|}{ SPI-8 amplicons } & \multicolumn{3}{|c|}{ SPI-10 amplicons } \\
\hline & & a & $\mathbf{b}$ & $\mathbf{a}$ & $\mathbf{b}$ & c \\
\hline MTCC & Typhimurium MTCC 98 & - & - & - & - & - \\
\hline MTCC & Paratyphi A MTCC 735 & + & + & + & + & + \\
\hline MTCC & Bovismorbificans MTCC 1162 & - & - & - & - & - \\
\hline MTCC & Brunei MTCC 1168 & - & - & - & - & - \\
\hline MTCC & S. enterica subsp. arizonae MTCC 660 & - & - & - & - & - \\
\hline MTCC & Virchow MTCC 1163 & - & - & - & - & - \\
\hline MTCC & Weltevreden MTCC 1169 & - & - & - & - & - \\
\hline Water/blood & Typhi $(15 / 14)$ & + & + & + & + & + \\
\hline Fish & Typhi (12) & + & + & - & - & - \\
\hline Water/fish/sprouts & Typhimurium (11/6/22) & - & - & - & - & - \\
\hline Water & Typhimurium (3) & + & + & + & + & + \\
\hline Water/blood & Paratyphi A (6/2) & + & + & + & + & + \\
\hline Blood & Paratyphi B (1) & - & - & - & - & - \\
\hline Blood & Paratyphi C (1) & - & - & - & - & - \\
\hline Fish & Worthington (12) & - & - & - & - & - \\
\hline Water & Enteritidis (3) & + & + & + & + & + \\
\hline Sprouts & Dublin (1) & - & - & - & - & - \\
\hline Sprouts & Washington (1) & + & + & + & + & + \\
\hline
\end{tabular}

${ }^{\star}$ The numbers of serovars from the respective sources are shown in parentheses.

Dublin, Enteritidis and Gallinarum. It has been predicted that the fimbrial operon on SPI-10 may play a role in determining host specificity for Salmonella serovar Typhi. However, our studies demonstrated that it was also present in Salmonella serovars Typhimurium and Washington. It was present in all isolates belonging to Salmonella serovars Typhi and Paratyphi A except for serovar Typhi isolated from fish (Table 1), and was absent in Salmonella serovars Worthington, Dublin, Paratyphi B and Paratyphi C. Isolates belonging to Salmonella serovar Typhimurium obtained from sprouts and fish were negative for SPI-10, but isolates from water contained SPI-10. Salmonella isolates belonging to serovar Enteritidis isolated from water were also positive. Both SPI-8 and SPI-10 were absent in Salmonella serovars Typhimurium MTCC 98, Bovismorbificans MTCC 1162, Brunei MTCC 1168, Virchow MTCC 1163 and Weltevreden MTCC 1169 and in S. enterica subsp. arizonae MTCC 660.

Interestingly, Salmonella serovar Typhi isolated from fish only possessed SPI-8, whilst all other Salmonella serovar Typhi were positive for both of the PAIs as predicted. However, three Salmonella serovar Typhimurium, three Salmonella serovar Enteritidis and one Salmonella serovar Washington were positive for both SPI-8 and SPI-10. A significant observation was that none of the isolates was negative for SPI- 8 and positive for SPI-10, giving a strong indication that whenever SPI-10 occurs, SPI-8 is also present. Genotyping data by PFGE analysis has shown that Salmonella serovar Typhimurium isolates with differences in PAI distribution are from different clones (Saroj et al., 2008).
SPI-8 and SPI-10 were detected in food (sprouts) and environmental (water) isolates in addition to the clinical (blood) isolates, demonstrating the wide distribution of SPI8 and SPI-10 among Salmonella serovars. This could be due to horizontal gene transfer taking place in Salmonella. Acquisition of virulence genes by horizontal gene transfer in Salmonella has contributed to the emergence of new pathovars and the acquisition of factors that extend their host range (Rabsch et al., 2002). Serovars that showed the absence of SPI-8 were negative for both of the regions examined, whilst those that showed the absence of SPI-10 were negative for all three regions examined. Serovars showing the presence of SPI- 8 and SPI-10 were positive for all of the regions examined. In addition, sequencing of PCR products from Salmonella serovars Typhi, Typhimurium, Paratyphi A, Washington and Enteritidis show that these regions are highly conserved ( $100 \%$ identity). This observation is particularly significant as these regions (SPI- 8 and SPI-10) were conserved among the Salmonella isolates from India, isolates from the distant geographical region of Nepal and also in the sequenced Salmonella serovars. This clearly suggests that these PAIs may have been acquired before Salmonella species evolved into different serovars. Gain or loss of these islands depends on the environmental niche occupied, as three isolates belonging to Salmonella serovar Typhimurium obtained from water showed the presence of both islands, whilst in other isolates these regions were absent. Similarly, Salmonella serovar Typhi isolated from fish has lost SPI-10.

The presence and absence of SPI-8 and SPI-10 in Salmonella serovar Typhimurium, Washington and Typhi 
(from fish) did not correlate with the expected results from the genome sequence data available in GenBank. To check the authenticity of these serovars, serotyping results were confirmed using antisera from Germany and molecular serotyping was carried out as described by Hirose et al. (2002) and Agron et al. (2001).

The results matched the previous serotyping results. None of the Typhimurium or Washington isolates reacted with antisera against Typhi, and PCR results correlated well with the serology (Supplementary Fig. S2).

Genome-based studies have uncovered a set of Salmonella genes that is restricted among these organisms and which may be important in deciding their host specificities. However, their predictions regarding the distribution of SPI- 8 and SPI-10 are incorrect. Our results showed that SPI- 8 and SPI-10 are widely distributed among Salmonella serovars. The exact role of each of these SPIs and their distribution need to be studied further to understand the host specificity and invasiveness of Salmonella serovars. Comparative genomics requires input from multiple genomic sequences; however, complete genome sequences of only a few bacteria have been completed to date. Therefore, there is a need to conduct laboratory experiments to support predictions made by genome database analysis and routine characterization of food and environmental isolates for pathogenicity-related genes from a public health point of view.

\section{ACKNOWLEDGEMENTS}

We thank Dr B. P. Kapadnis, University of Pune, India, for providing Salmonella isolates from water and blood.

\section{REFERENCES}

Agron, P. G., Walker, R. L., Kinde, H., Sawyer, S. J., Hayes, D. C., Wollard, J. \& Andersen, G. L. (2001). Identification by subtractive hybridization of sequences specific for Salmonella enterica serovar Enteritidis. Appl Environ Microbiol 67, 4984-4991.

Al-Khaldi, S. F. \& Mossoba, M. M. (2004). Gene and bacterial identification using high throughput technologies: genomics, proteomics, and phonemics. Nutrition 20, 32-38.

Baird-Parker, A. C. (1990). Foodborne salmonellosis. Lancet 336, 1231-1235.

Bandekar, J. R., Kamat, A. S., Karani, M., Dhokane, V., Shashidhar, R., Kakatkar, A., Ghadge, N., Bhat, A., Venugopal, V. \& Warrier, S. B. (2004). Bacteriological quality of farmed freshwater fish and shellfish meant for export. Fishery Technol 41, 57-62.

Baumler, A. J., Tsolis, R. M., Ficht, T. A. \& Adams, L. G. (1998). Evolution of host adaptation in Salmonella enterica. Infect Immun 66, 4579-4587.

Bhatta, D. R., Bangtrakulnonth, A., Tishyadhigama, P., Saroj, S. D., Bandekar, J. R., Hendriksen, R. S. \& Kapadnis, B. P. (2007). Serotyping, PCR, phage-typing and antibiotic sensitivity testing of
Salmonella serovars isolated from urban drinking water supply systems of Nepal. Lett Appl Microbiol 44, 588-594.

Chan, K., Baker, S., Kim, C. C., Detweiler, C. S., Dougan, G. \& Falkow, S. (2003). Genomic comparison of Salmonella enterica serovars and Salmonella bongori by use of an S. enterica serovar Typhimurium DNA microarray. J Bacteriol 185, 553-563.

Chiu, C. H., Tang, P., Chu, C., Hu, S., Bao, Q., Yu, J., Chou, Y., Wang, H. \& Lee, Y. (2005). The genome sequence of Salmonella enterica serovar Choleraesuis, a highly invasive and resistant zoonotic pathogen. Nucleic Acids Res 33, 1690-1698.

Dhokane, V. S., Hajare, S., Shashidhar, R., Sharma, A. \& Bandekar, J. R. (2006). Radiation processing to ensure safety of minimally processed carrot (Daucus carota) and cucumber (Cucumis sativus): optimization of dose for the elimination of Salmonella Typhimurium and Listeria monocytogenes. J Food Prot 69, 444-448.

Edwards, R. A., Olsen, G. J. \& Maloy, S. R. (2002). Comparative genomics of closely related salmonellae. Trends Microbiol 10, 94-99.

Groisman, E. A. \& Ochman, H. (1996). Pathogenicity islands: bacterial evolution in quantum leaps. Cell 87, 791-794.

Hensel, M. (2004). Evolution of pathogenicity islands of Salmonella enterica. Int J Med Microbiol 294, 95-102.

Hirose, K., Itoh, K. I., Nakajima, H., Kurazono, T., Yamaguchi, M., Moriya, K., Ezaki, T., Kawamura, Y., Tamura, K. \& Watanabe, H. (2002). Selective amplification of $t y v(r f b E)$, prt ( $r f b s)$, viaB, and fliC genes by multiplex PCR for identification of Salmonella enterica serovar Typhi and Paratyphi A. J Clin Microbiol 40, 633-636.

Lynch, M., Painter, J., Woodruff, R., Braden, C. \& Centers for Disease Control and Prevention (2006). Surveillance for foodborne-disease outbreaks - United States, 1998-2002. MMWR Surveill Summ 55, 1-42.

Oelschlaeger, T. A. \& Hacker, J. (2004). Impact of pathogenicity islands in bacterial diagnostics. APMIS 112, 930-936.

Plym-Forshell, L. \& Wierup, M. (2006). Salmonella contamination: a significant challenge to the global marketing of animal food products. Rev Sci Tech 25, 541-554.

Rabsch, W., Andrews, H. L., Kingsley, R. A., Prager, R., Tschape, H., Adams, L. G. \& Baumler, A. J. (2002). Salmonella enterica serotype Typhimurium and its host-adapted variants. Infect Immun 70, 2249-2255.

Saroj, S. D., Shashidhar, R., Dhokane, V., Hajare, S., Sharma, A. \& Bandekar, J. R. (2006). Microbiological evaluation of sprouts marketed in Mumbai, India, and its suburbs. J Food Prot 69, 2515-2518.

Saroj, S. D., Shashidhar, R. \& Bandekar, J. R. (2008). Genotypic characterization of Salmonella enterica serovar Typhimurium isolated from sprouts and fish. Food Sci Tech Int (in press).

Schmidt, H. \& Hensel, M. (2004). Pathogenicity islands in bacterial pathogenesis. Clin Microbiol Rev 17, 14-56.

Townsend, S. M., Kramer, N. E., Edwards, R., Baker, S., Hamlin, N., Simmonds, M., Stevens, K., Malony, S., Parkhill, J. \& other authors (2001). Salmonella enterica serovar Typhi possesses a unique repertoire of fimbrial gene sequences. Infect Immun 69, 2894-2901.

Vernikos, G. S. \& Parkhill, J. (2006). Interpolated variable order motifs for identification of horizontally acquired DNA: revisiting the Salmonella pathogenicity islands. Bioinformatics 22, 2196-2203.

Wilson, J. W., Schurr, M. J., LeBlanc, C. L., Ramamurthy, R., Buchanan, K. L. \& Nickerson, C. A. (2002). Mechanisms of bacterial pathogenicity. Postgrad Med J 78, 216-224. 